\title{
Steepest descent on real flag manifolds
}

\author{
J.-H. Eschenburg and A.L. Mare
}

10th November 2018

\section{Introduction}

Among the compact homogeneous spaces, a very distinguished subclass is formed by the (generalized) real flag manifolds which by definition are the orbits of the isotropy representations of Riemannian symmetric spaces $(s-$ orbits). This class contains most compact symmetric spaces (e.g. all hermitian ones), all classical flag manifolds over real, complex and quaternionic vector spaces, all adjoint orbits of compact Lie groups (generalized complex flag manifolds) and many others. They form the main examples for isoparametric submanifolds and their focal manifolds (so called constant principal curvature manifolds); in fact for most codimensions these are the only such spaces (cf. [7], [9], [8]).

Any real flag manifold $M$ enjoys two very peculiar geometric properties: It carries a transitive action of a noncompact Lie group $G$, and it is embedded in euclidean space as a taut submanifold, i.e. almost all height or coordinate functions are perfect Morse functions (at least for $\mathbb{Z} / 2$-coefficients). The aim of our paper is to link these two properties by the following theorem: The gradient flow of any height function is a one-parameter subgroup of $G$ where the gradient is defined with respect to a suitable homogeneous metric $s$ on $M$; in the case where $M$ is an adjoint orbit $s$ is a homogeneous Kähler metric. In other words, the lines of steepest descend for the height function (gradient flow lines) are obtained by applying a one-parameter subgroup of $G$. This is an elementary fact when $M$ is a euclidean sphere and $G$ its conformal group: The gradient of any height function is a conformal vector field. For adjoint orbits, the (generalized) complex flag manifolds, this fact was observed earlier by Guest and Ohnita [4]. Our more general result can be derived from their theorem since real flag manifolds are contained in complex flag manifolds 
as fixed point sets under certain involutions. However a short direct proof might be desirable. We would like to thank Martin Guest and Peter Quast for hints and discussion.

\section{Root space decomposition}

Let $P=G / K$ be a symmetric space of noncompact type where $G$ is a connected noncompact semisimple Lie group and $K \subset G$ a maximal compact subgroup (cf. [6]). Let $\sigma$ be the corresponding involution on $G$ with fixed point set $K$. Consider the corresponding Cartan decomposition

$$
\mathfrak{g}=\mathfrak{k} \oplus \mathfrak{p}
$$

where $\mathfrak{g}$ and $\mathfrak{k}$ denote the Lie algebras of $G$ and $K$ and $\mathfrak{p}$ the (-1)-eigenspace of (the differential of) $\sigma$. The adjoint action of $K$ leaves $\mathfrak{p}$ invariant; this is the isotropy representation of $P$. As usual we consider an $\operatorname{Ad}(G)$-invariant indefinite inner product $b$ on $\mathfrak{g}$ with $b>0$ on $\mathfrak{p}$ and $b<0$ on $\mathfrak{k}$ (e.g. the Killing form) and define a positive definite inner product $\langle$,$\rangle on \mathfrak{g}$ which is $b$ on $\mathfrak{p}$ and $-b$ on $\mathfrak{k}$, and such that $\mathfrak{p} \perp \mathfrak{k}$. Then any $\operatorname{ad}(x)$ with $x \in \mathfrak{p}$ is self adjoint on $\mathfrak{g}$. Hence a maximal abelian subspace $\mathfrak{a} \subset \mathfrak{p}$ gives rise to a family of mutually commuting selfadjoint endomorphisms ad $(x)$ of $\mathfrak{g}$ where $x \in \mathfrak{a}$. These have a common eigenspace decomposition

$$
\mathfrak{g}=\sum_{\alpha \in \hat{R}} \mathfrak{g}_{\alpha}, \quad \mathfrak{g}_{\alpha}:=\{z \in \mathfrak{g} ;[x, z]=\alpha(x) z \forall x \in \mathfrak{a}\}
$$

where $\hat{R} \subset \mathfrak{a}^{*}$ is the set of roots including 0 .

After fixing some arbitrary $x \in \mathfrak{a}$, there are three disjoint subsets of $\hat{R}$, formed by the roots $\alpha$ with $\alpha(x)>0, \alpha(x)=0$ and $\alpha(x)<0$, respectively. Hence we have the decomposition

$$
\mathfrak{g}=\mathfrak{n}_{+} \oplus \mathfrak{c} \oplus \mathfrak{n}_{-}
$$

with

$$
\mathfrak{n}_{+}=\sum_{\alpha(x)>0} \mathfrak{g}_{\alpha}, \quad \mathfrak{c}=\sum_{\alpha(x)=0} \mathfrak{g}_{\alpha}, \quad \mathfrak{n}_{-}=\sum_{\alpha(x)<0} \mathfrak{g}_{\alpha} .
$$

Since $\sigma=-i d$ on $\mathfrak{a}$, we have

$$
\left(\mathfrak{g}_{\alpha}\right)^{\sigma}=\mathfrak{g}_{-\alpha},
$$


hence $\sigma$ interchanges $\mathfrak{n}_{+}$and $\mathfrak{n}_{-}$. Any $a \in \mathfrak{g}$ allows a unique decomposition $a=a_{-}+a_{o}+a_{+}$with $a_{ \pm} \in \mathfrak{n}_{ \pm}$and $a_{o} \in \mathfrak{c}$, and if $a \in \mathfrak{k}$ or $a \in \mathfrak{p}$, we have $a_{-}=a_{+}^{\sigma}$ or $a_{-}=-a_{+}^{\sigma}$, respectively.

\section{Generalized real flag manifolds}

By definition, generalized real flag manifolds are the orbits of the isotropy representation of a symmetric space $P=G / K$ of noncompact type. We consider an isotropy orbit $M \subset \mathfrak{p}$. For any fixed $x \in M$ we have $M=$ $\operatorname{Ad}(K) x$. The stabilizer subgroup is

$$
S=\{k \in K ; \operatorname{Ad}(k) x=x\},
$$

thus $M$ may be identified with the coset space $K / S$ by $k S \mapsto \operatorname{Ad}(k) x$.

Now choose a maximal abelian subalgebra $\mathfrak{a} \subset \mathfrak{p}$ containing $x$. Such $\mathfrak{a}$ is uniquely determined up to applying $\operatorname{Ad}(s)$ with $s \in S$; this fact is just the conjugacy of maximal flat subspaces in the symmetric subspace $\tilde{P}=C / S \subset P$ where $C$ (with Lie algebra $\mathfrak{c}$ ) is the centralizer of $x$ in $G{ }^{1}$ Note that each $\operatorname{Ad}(s), s \in S$, commutes with $\operatorname{ad}(x)$ and thus preserves the eigenspaces of $\operatorname{ad}(x)$, hence $\mathfrak{n}_{-}=\sum_{\alpha(x)<0} \mathfrak{g}_{\alpha}$ is also invariant under $\operatorname{Ad}(s)$.

Next we show that the natural $K$-action on $M$ can be extended to a $G$-action. Consider

$$
H=\left\{g \in G ; \operatorname{Ad}(g)\left(x+\mathfrak{n}_{-}\right)=x+\mathfrak{n}_{-}\right\}
$$

which is a closed subgroup of $G$ containing $S$ as a subgroup. One can see that the Lie algebra of $H$ is $\mathfrak{h}=\mathfrak{c}+\mathfrak{n}_{-}$. Note that $H$ does not depend on the choice of $\mathfrak{a}$. Indeed, if instead of $\mathfrak{a}$ we start our construction with $\mathfrak{a}^{\prime}=\operatorname{Ad}(s) \mathfrak{a}$, where $s \in S$, then $\mathfrak{n}_{-}^{\prime}=\operatorname{Ad}(s) \mathfrak{n}_{-}=\mathfrak{n}_{-}$, and we end up with $H^{\prime}=H$.

Lemma $3.1 K$ acts transitively on the coset space $G / H$ with stabilizer $S$. Hence $G / H$ can be identified with $K / S=M$.

Proof. Let $M^{\prime} \subset G / H$ be the orbit of $e H \in G / H$ under the subgroup $K \subset G$. We show first that it is open in $G / H$. To see this it suffices to show

\footnotetext{
${ }^{1}$ Geometrically, $\tilde{P}$ is the union of all geodesics parallel to the geodesic $\exp (\mathbb{R} x) \subset P$ which is obviously invariant under geodesic reflection, hence it is a symmetric subspace.
} 
$\mathfrak{k}+\mathfrak{h}=\mathfrak{g}$, i.e. one needs to show $\mathfrak{g}_{\alpha} \subset \mathfrak{k}+\mathfrak{h}$ for each $\alpha \in R$ with $\alpha(x)>0$. In fact, take $z \in \mathfrak{g}_{\alpha}$, decompose it as $z=v+u$ with $v \in \mathfrak{p}$ and $u \in \mathfrak{k}$ and notice that $z^{\sigma}=-v+u \in \mathfrak{g}_{-\alpha} \subset \mathfrak{h}$. Thus the $K$-orbit $M^{\prime}$ is open. But it is also closed in $G / H$ since $K$ is compact. So $M^{\prime}$ coincides with $G / H$.

The $K$-stabilizer of $e H \in G / H$ is $K \cap H$; we have to show $K \cap H=S$. Clearly $S \subset K \cap H$. Vice versa, if $k \in K \cap H$, then

$$
\operatorname{Ad}(k) x-x \in \mathfrak{p} \cap \mathfrak{n}_{-} .
$$

But $\mathfrak{p} \cap \mathfrak{n}_{-}=0$, because if $z$ belongs to this intersection, then $z^{\sigma}=-z \in \mathfrak{n}_{+}$, hence $z \in \mathfrak{n}_{-} \cap \mathfrak{n}_{+}=0$. We deduce that $\operatorname{Ad}(k) x=x$, which means $k \in S$.

Thus the action of $G$ on $G / H=K / S=M$ is an extension of the $K$ action. $^{2}$ We will denote this action $G \times M \rightarrow M$ by $(g, x) \mapsto g . x$. When restricted to $k \in K \subset G$ we have $k . x=\operatorname{Ad}(k) x$. Similarly, the infinitesimal action $\mathfrak{g} \times M \rightarrow T M$ will be denoted by $(a, x) \mapsto a \cdot x:=\left.\frac{d}{d t}\right|_{t=0} \exp (t a) \cdot x$ where $a \in \mathfrak{g}$, and $a . x=[a, x]$ whenever $a \in \mathfrak{k}$.

\section{Steepest descend}

Theorem 4.1 Let $G / K$ be a symmetric space of noncompact type, $\mathfrak{g}=\mathfrak{k}+\mathfrak{p}$ the corresponding Cartan decomposition and $M \subset \mathfrak{p}$ a real flag manifold (isotropy orbit). Let $q \in \mathfrak{p}$ and $f: M \rightarrow \mathbb{R}, f(x)=\langle q, x\rangle$. Then there is a $K$-invariant Riemannian metric $s: T M \rightarrow T^{*} M$ on $M$ such that the flow lines $x(t)$ of the s-gradient $\nabla^{s} f=s^{-1} d f$ satisfy

$$
x(t)=\exp (-t q) \cdot x(0)
$$

Proof. Choose an arbitrary $x \in M$ and consider the decomposition (3) corresponding to $x$. We have to show that $\nabla^{s} f(x)=-q . x$. To compute $q . x$ we look for $r \in \mathfrak{k}$ with $q . x=r . x$, i.e. $q-r \in \mathfrak{h}$. We have $q=q_{o}+\sum_{\alpha(x)>0} q_{\alpha}$

\footnotetext{
${ }^{2}$ There is a more geometric description of this action: Consider $\mathfrak{p}$ as the tangent space of $P=G / K$ at some base point $o \in P$. We may project any nonzero $x \in T_{o} P$ to the infinite boundary $P(\infty)$ (mind that $P$ is a simply connected space of nonpositive curvature) by the map $\pi_{\infty}(x)=\gamma_{x}(-\infty)$ where $\gamma_{x}$ is the geodesic in $P$ starting at $o$ with initial vector $x$. The isometry group $G$ acts on $P(\infty)$ and leaves $\pi_{\infty}(M)$ invariant; this is the $G$-action. See [1] for details.
} 
with $q_{o} \in \mathfrak{c}$ and $q_{\alpha}=z_{\alpha}-z_{\alpha}^{\sigma}$ for some $z_{\alpha} \in \mathfrak{g}_{\alpha}$. We may assume $q_{o}=0$ since $q_{o} . x=0$. Then we put $r=\sum_{+}\left(z_{\alpha}+z_{\alpha}^{\sigma}\right) \in \mathfrak{k}$ and hence $r-q=2 \sum_{+} z_{\alpha}^{\sigma} \in \mathfrak{h}$ where $\sum_{+}$always denotes $\sum_{\alpha(x)>0}$. Now

$$
r . x=[r, x]=-a d(x) \sum_{+}\left(z_{\alpha}+z_{\alpha}^{\sigma}\right)=-\sum_{+} \alpha(x)\left(z_{\alpha}-z_{\alpha}^{\sigma}\right)=-\sum_{+} \alpha(x) q_{\alpha} .
$$

Any $v \in T_{x} M$ has a decomposition $v=\sum_{+} v_{\alpha}$ with $v_{\alpha} \in\left(\mathfrak{g}_{\alpha}+\mathfrak{g}_{-\alpha}\right) \cap \mathfrak{p}$ for $\alpha(x)>0$, and our metric $s$ on $T_{x} M$ will be of the form

$$
\langle v, w\rangle_{s}=\sum_{+} s_{\alpha}\left\langle v_{\alpha}, w_{\alpha}\right\rangle
$$

for certain numbers $s_{\alpha}>0$. We have to choose $s$ such that for all $v \in T_{x} M$

$$
\left\langle\nabla^{s} f(x), v\right\rangle_{s}=-\langle r . x, v\rangle_{s} .
$$

The left hand side is

$$
\left\langle\nabla^{s} f(x), v\right\rangle_{s}=d f_{x} v=\langle q, v\rangle=\sum_{+}\left\langle q_{\alpha}, v_{\alpha}\right\rangle
$$

while the right hand side is

$$
-\langle r . x, v\rangle_{s}=\sum_{+} s_{\alpha} \alpha(x)\left\langle q_{\alpha}, v_{\alpha}\right\rangle .
$$

Hence we obtain the result by putting

$$
s_{\alpha}=1 / \alpha(x)
$$

\section{$5 \quad$ Extrinsic symmetric spaces}

An extrinsic symmetric space is a submanifold $M$ in euclidian space such that $M$ is preserved by the reflections at all of its (affine) normal spaces. By a result of Ferus [3] (also cf. [2]), after splitting off euclidean factors, $M$ has precisely the form of an s-orbit $M=\operatorname{Ad}(K) x_{o} \subset \mathfrak{p}$ where $\mathfrak{p}$ corresponds to a symmetric space $P=G / K$ and where $x_{o} \in \mathfrak{p}$ satisfies

$$
\alpha\left(x_{o}\right) \in\{-1,0,1\}
$$

for all $\alpha \in R$. In this case the metric $s$ of Theorem 4.1 agrees to the given inner product $\langle$,$\rangle (cf. (9)). By applying Theorem 4.1$ one obtains: 
Theorem 5.1 If $M=\operatorname{Ad}(K) x_{o} \subset \mathfrak{p}$ is extrinsic symmetric, then the gradient lines of the height function $h(x)=\langle q, x\rangle$ with respect to the metric $\langle$, are of the form

$$
x(t)=\exp (-t q) \cdot x(0)
$$

\section{Adjoint orbits}

In the particular case of complex flag manifolds (i.e. adjoint orbits of compact Lie groups) we will establish relations between Theorem 4.1 and previously known results.

Let $K$ be a compact semisimple Lie group of Lie algebra $\mathfrak{k}$, and $T \subset K$ a maximal torus of Lie algebra $\mathfrak{t}$. Consider the adjoint orbit $M=\operatorname{Ad}(K) x$ for $x \in \mathfrak{k}$. If $G=K^{\mathbb{C}}$ is the complexification of $K$, then $G / K$ is a non-compact symmetric space and

$$
\mathfrak{g}=\mathfrak{k}+\mathfrak{i k}
$$

is a Cartan decomposition of $\mathfrak{g}=\operatorname{Lie}(G)=\mathfrak{k} \otimes \mathbb{C}$ (the involution $\sigma$ is just the complex conjugation). Since $M$ is (up to a multiple of i) an isotropy orbit of $G / K$, the results of the previous section can be applied here, too. The goal of this section is to point out that the metric on $M$ for which the lines of steepest descent are orbits of one-parameter subgroups of $G$ is well known: It is the Kähler metric (cf. [4], [3]).

It is well known that any adjoint orbit $M=\operatorname{Ad}(K) x$ is a complex manifold. In fact, in the language of Section 4 we have $M=G / H$ but here $G$ and $H$ are complex Lie groups and hence $M$ is a complex manifold. The corresponding complex structure $J$ on $T_{x} M$ can be described as follows. Choose a maximal abelian subalgebra $\mathfrak{t} \subset \mathfrak{k}$ with $x \in \mathfrak{t}$. The corresponding roots are considered as real linear forms $\alpha \in \mathfrak{t}^{*}$ while the eigenvalues of $\operatorname{ad}(x)$ are purely imaginary, $\mathrm{i} \alpha(x)$. Let $\mathfrak{k}_{\alpha} \subset \mathfrak{k} \otimes \mathbb{C}$ be the root spaces. Then

$$
T_{x} M=\sum_{\alpha(x)>0} \mathfrak{k}_{\alpha}^{r}
$$

where $\mathfrak{k}_{\alpha}^{r}=\left(\mathfrak{k}_{\alpha}+\mathfrak{k}_{-\alpha}\right) \cap \mathfrak{k}$ is the real root space. Now $J$ leaves invariant each $\mathfrak{k}_{\alpha}^{r}$ and on $\mathfrak{k}_{\alpha}^{r}$ it is a multiple of $\operatorname{ad}(x)$ :

$$
\operatorname{ad}(x)=\alpha(x) J
$$


The second ingredient for the Kähler metric is the Kähler form $\omega$ which is defined as follows: If $v=\operatorname{ad}(a) x$ and $w=\operatorname{ad}(b) x$ are tangent vectors of $M$ at the point $x$, then

$$
\omega_{x}(v, w):=\langle x,[a, b]\rangle=\langle[x, a], b\rangle=\langle v, b\rangle=\left\langle v, \operatorname{ad}(x)^{-1} w\right\rangle
$$

where $\langle$,$\rangle denotes an A d(K)$-invariant inner product on $\mathfrak{k}$.

Now the Kähler metric ( , ) on $T_{x} M$ is defined as follows. For $v, w \in T_{x} M$ we have

$$
(v, w)=\omega_{x}(v, J w) .
$$

Hence from (10) and (11) we obtain

$$
(v, w)=\omega_{x}(v, J w)=\left\langle v, \operatorname{ad}(x)^{-1} J w\right\rangle=\frac{1}{\alpha(x)}\langle v, w\rangle=\langle v, w\rangle_{s}
$$

(cf. (9)). Since $\mathfrak{p}=$ ik in the present case, we obtain from Theorem 4.1:

Theorem 6.1 (cf. [4], [3]) Let $K$ be a compact Lie group and $M=\operatorname{Ad}(K) x_{0}$ $\subset \mathfrak{k}$ an adjoint orbit, equipped with its Kähler metric (, ) as in (12) and acted on by the complexified group $G=K^{\mathbb{C}}$ as described above. Let $\langle$,$\rangle be$ the corresponding $\operatorname{Ad}(K)$-invariant inner product on $\mathfrak{k}$. Then for any $q \in \mathfrak{k}$ the gradient lines $x(t)$ of the function $f: M \rightarrow \mathbb{R}, f(x)=\langle q, x\rangle$, are orbits of a 1-parameter subgroup of $G=K^{\mathbb{C}}$, namely

$$
x(t)=\exp (\mathrm{i} t q) \cdot x(0) .
$$

\section{References}

[1] W. Ballmann, M. Gromov, V. Schroeder: Manifolds of Nonpositive Curvature, Birkhäuser 1985

[2] J.-H. Eschenburg, E. Heintze, Extrinsic symmetric spaces and orbits of s-representations, Manuscripta math. 88 (1995), 517 - 524.

[3] D. Ferus, Symmetric submanifolds of euclidean space, Math. Ann. 247 (1980), 81-93

[4] M. A. Guest, Y. Ohnita, Group actions and deformations for harmonic maps, J. Math. Soc. Japan, 45 1993, 671-704 
[5] M. A. Guest, Harmonic Maps, Loop Groups and Integrable Systems, LMS Student Texts 38, Cambridge Univ. Press, 1997

[6] S. Helgason: Differential Geometry, Lie groups and Symmetric Spaces, Academic Press 1978

[7] E. Heintze, C. Olmos, G. Thorbergsson: Submanifolds with constant principal curvatures and normal holonomy group, Int. J. Math. 2 (1991), $167-185$

[8] C. Olmos: Isoparametric submanifolds and their homogeneous structure, J. Diff. Geom. 38 (1993), 225 - 234

[9] G. Thorbergsson: Isoparametric foliations and their buildings, Ann. Math. 133 (1991), 429 - 446 